\title{
Improving the way to calculate risks: the qualitative and quantitative risk index $\mathbf{R}^{*}$
}

\author{
G. Reniers ${ }^{1,2,3}$ \\ ${ }^{1}$ Safety and Security Science Group, Faculty of TPM, \\ Delft University of Technology, The Netherlands \\ ${ }^{2}$ ARGoSS, FTEW, University of Antwerp, Belgium \\ ${ }^{3}$ CEDON, FE\&M, KULeuven, Belgium
}

\begin{abstract}
This paper takes the viewpoint that the current rational approach that is used throughout the world to calculate risks, is insufficient for decision-making and dealing with risks in the next generations. Moral aspects and the acceptability of risks should be taken into consideration while determining risk priorities to optimize decision-making. A risk calculation formula is proposed to this end, whereby principles such as voluntariness, equity and fairness are taken into account in the formula.
\end{abstract}

Keywords: risk calculation, moral aspects of risk, acceptability of risk.

\section{Introduction}

As defined by the Center for Chemical Process Safety [1], operational risk can be seen as an index of potential economic loss, human injury, or environmental damage, that is measured in terms of both the incident probability and the magnitude of the loss, injury or damage. The operational risk associated with a specific unwanted event can thus be expressed as the product of two factors: the likelihood that the event will occur $\left(L_{\text {event }}\right)$ and its consequences $\left(C_{\text {event }}\right)$. Therefore, such an operational risk index represents the 'expected consequence' of the undesired event:

$$
R_{\text {event }}=L_{\text {event }} C_{\text {event }}
$$

However, the risk estimation always refers to specific scenarios in which also the perception and the attitude to the consequences of the decision-maker may 
differ importantly. For example, most people judge a HILP (high impact low probability) event as more undesirable than a LIHP (low impact high probability) event, even if the expected consequence of the two events are exactly the same. By introducing a risk preference parameter, the previously formulated risk index, taking into account decision-makers' preferences, can be reformulated into:

$$
R_{\text {event }}=L_{\text {event }}\left(C_{\text {event }}\right)^{a}
$$

where the parameter ' $a$ ' represents the attitude of the decision-maker to the consequences. If a decision-maker is consequence-averse (or also called 'riskaverse'), $a>1$; if he is risk-neutral, $a=1$, and if he is risk-seeking, $a<1$. It is obvious from the previous that the way a risk is calculated, which depends on preferences of people, has an influence on the resulting index outcomes. The risk index should thus be seen as the 'calculated perception of risk reality' by a person or a group of persons using a certain calculation method that they agreed upon. In any case, the index outcomes allow to distinguish between different types and kinds of risks.

However, as mentioned by van de Poel and Fahlquist [2], psychological literature on risk perception has established that lay people include contextual elements in how they perceive and understand risks (e.g. [3]). Such contextual elements are for instance voluntariness, familiarity, dread, exposure, catastrophic potential, controllability, perceived benefits, and time delay (future generations). The fact that there is a difference in risk perception between lay people and experts, is often interpreted as the result of irrationality of the lay people. However, such an interpretation indirectly assumes that the expert opinion, or the technical conceptualization of risk, is the right one and that lay people should just be educated to gain the same viewpoint. However, for example Slovic [3] and Roeser $[4,5]$ explain that the contextual elements considered by lay people are actually relevant for a better perception of reality of risk, and are thus important for the acceptability of risks and for adequately managing risks.

\section{Moral aspects and acceptability of risks}

Sunstein [6] mentions that when asked to assess the risks and benefits associated with certain items, people tend to think that hazardous activities contain low benefits, whereas beneficial activities contain low hazards. Rarely, people consider an activity to be both highly beneficial and hazardous, or to be both benefit-free and hazard-free. Slovic [7] even suggests a so-called "affect heuristic", by which people have mainly an emotional reaction to certain situations, processes, activities, products, etc. Hence, from these observations it is possible to conclude that emotions and feelings are essential elements to consider when dealing with operational risks and appreciating operational safety. Besides objective consequences and probabilities of potential scenarios, moral aspects (possibly fed by emotions), should thus play an important role in the calculation of safety. The subsequent activities of hazard identification, risk analysis (including estimation/calculation of consequences and likelihood), risk evaluation and risk prioritization, a process known as risk assessment, only serves the purpose to have a rational idea of all the prioritized (rational) risks (for a certain scope of 
the safety study), in order to make decisions about what risks to deal with first, second, etc. (hence, safety budget allocations!). However, to improve the safety budget allocation decision process, not merely rational risk prioritizations should be carried out, but rather safety appreciation prioritizations should be executed, where moral aspects are taken into account besides the rational aspects.

If moral aspects would be considered in the safety calculation process, economic analyses would perhaps be much less contested or debated, and more accepted as a valid approach to ensure that company policy is driven not by pure ratio or by pure emotion, but by a combination of both, and thus a full appreciation of all aspects of safety, rational as well as emotional, is strived for. The risk thermostat, originally developed by Wilde [8], and adapted by Adams [9-11], can be applied to an organization, and the importance of balanced safety appreciations becomes immediately obvious in this regard.

Everyone, also decision-makers in companies, have a propensity to take risks within appropriate limits. This propensity to take risks varies from one company to another, and it is influenced by the potential level of the rewards and losses arising from the organization's risk-taking decisions. Companies' so-called 'perceptions of risk' are determined by the safety appreciations carried out in the company. Furthermore, companies' risk policies represent a balancing act in which the perceptions of risk are balanced against the propensity to take risks. Benefits and losses arise from an organization taking risks, and the more risks (in general, thus also the more operational risks) an organization takes, the greater the potential level of costs and benefits.

If moral aspects are not taken into consideration in the safety calculation, and, as a result, the costs from the company's risk policy are (/may be) determined to be quite low (compared with the possible costs for the company if moral aspects would have been taken into account), the perception of a certain risk (e.g. a HILP risk) will be low, and the balance will tend towards risk-seeking behavior.

\section{A new approach to calculate risks: the Q\&Q Risk R*}

Literature (e.g. [12-15]) indicates the moral concerns that are often mentioned: voluntariness, the balance and distribution of benefits and risks (over different groups and over generations), and the availability of alternatives. These ethical dimensions can be used to enlarge the technical conception of operational risk, given in the introductory section, in an extended formula of a 'Qualitative and Quantitative' risk index, or a so-called Q\&Q risk index. The following formula for the Q\&Q Risk Index $\left(\mathrm{R}^{*}\right)$ is proposed to this end:

$$
R_{i}^{*}=\frac{\left(L_{i} \cdot C_{i}^{a}\right)}{\beta_{i} \cdot\left(E_{i} \cdot F_{i}^{b}\right)}=\frac{R_{i}}{\beta_{i} \cdot A_{i}}
$$

where: 


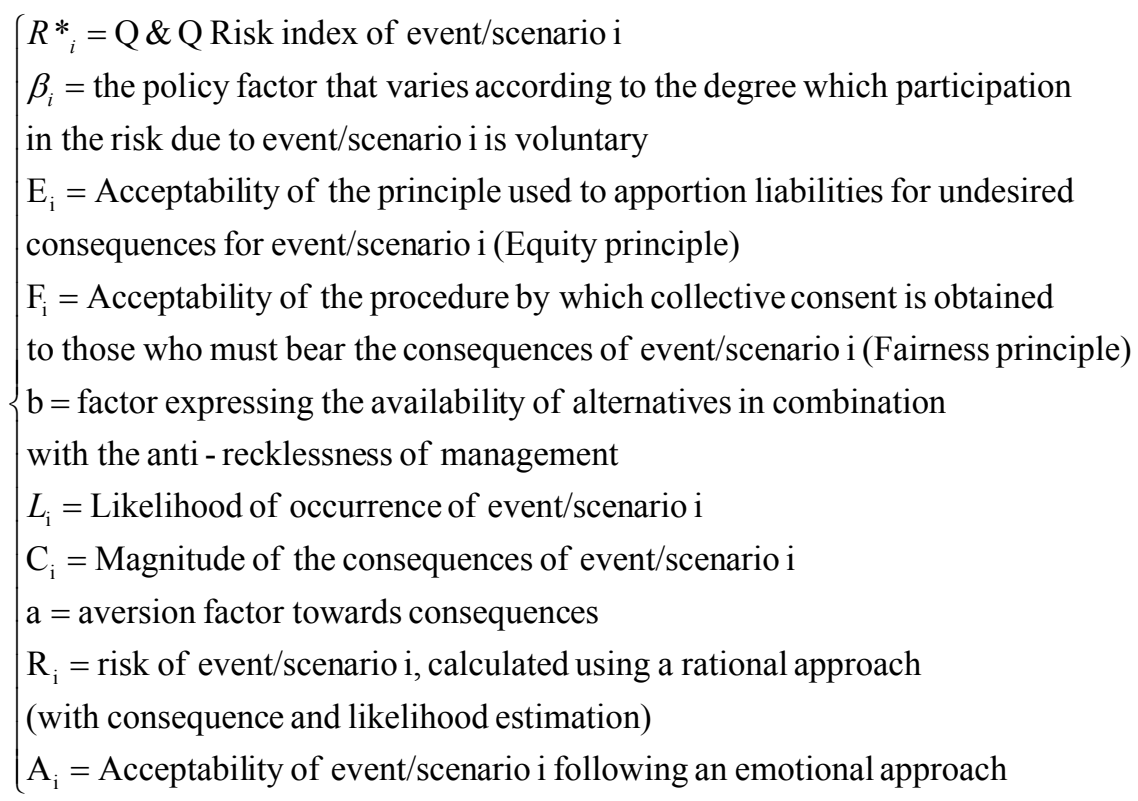

The factors $E$ and $F$ are also mentioned and defined by Rayner (1992) in an early attempt to extent the well-known rational risk formula " $R=L$. $C$ ". In the formula for an operational safety index that is proposed above, rationally calculated risk is combined with emotional aspects to be able to determine a proxy for operational safety within an organization. ' $E$ ' is a parameter representing the moral principle of 'Equity', taking the emotions concerning the balance and distribution of benefits and risks into consideration. ' $F$ ' is a parameter providing an idea of the moral principle of 'Fairness' or justification related with the operational risk of an event or scenario. Using this formula, the Q\&Q Risk index $\mathrm{R}^{*}$ of an undesired event is lower the more acceptable this event is, the higher the voluntariness of suffering the risk accompanying the event, and the lower the rationally calculated risk is. Since $\beta$ and $A$ are both dimensionless numbers, this $\mathrm{R}^{*}$ index is expressed in the same units as rational risk. Hence, if rational risk is expressed in monetary units per time units (e.g. €/year), $\mathrm{R}^{*}$ is also expressed in these units. The $\mathrm{R}^{*}$ could thus be used as an approach to calculate risk within an organisation, using both the well-known rational data such as probabilities, consequences, exposure, and the less-used moral principles such as fairness, equity, and recklessness of management.

If company management would desire to use this approach, it has to quantify the factors $\beta, E, F, a$ and $b$. Remark that the parameters $\beta, E$, and $F$ should be expressed as indices, and numbers for each of these parameters should be sufficiently different from each other to be relevant in the formula. A protocol within the company or within society should be designed to unambiguously determine the approach to quantify every parameter. Remark that if it would be possible that a parameter $\beta$, E, or $F$ could be chosen to be zero, consequently the 
$\mathrm{R}^{*}$ level for the event would be infinite, and thus the event can never be made safe enough, whatever the safety investments to influence (lower) the likelihood and/or consequences. Therefore, this possibility can best be excluded.

As an example, the following qualitative indices could be suggested for the parameters $\beta, E$, and $F$ :

- $\quad$ Very low: 0.1

- Low: 0.5

- Medium: 1.0

- High: 1.5

- Very high: 2.0

The parameters $a$ and $b$ are also suggested to be situated in the interval (0.2), to avoid stressing the aversion factor and the anti-recklessness factor too much in the risk formula.

\section{Illustrative example}

An illustrative example can be given to further explain the risk calculation formula. Assume that a comparison is needed for two accident scenarios, that is, a major industrial accident with one fatality within a single chemical plant, and a large-scale domino effect disaster within a cluster of chemical plants, affecting at least three companies. Assume we desire to know the difference in risk between both scenarios and taking moral principles into consideration. Table 1 provides the different (illustrative) quantitative $(L$ and $C)$ and qualitative $(a, b, E$, and $F)$ parameter values, for example determined by software (quantitative parameters) or by expert solicitation (qualitative parameters) for both scenarios. Since there are many people killed in the second (domino effect) scenario, the illustrative example sets the aversion factor $a$ equal to 2 in the disaster scenario. In the major accident scenario, people are assumed to be consequence averse, but only slightly.

Table 1: Parameter values for scenario 'major industrial accident' and for scenario 'domino effect disaster'.

\begin{tabular}{|l|l|}
\hline Major industrial accident & Domino effect disaster \\
\hline $\mathrm{L}=10^{-3}$ & $\mathrm{~L}=10^{-9}$ \\
\hline $\mathrm{C}=1$ fatality & $\mathrm{C}=10$ fatalities \\
\hline 1 fatality $=6,000,000 €$ & 1 fatality $=6,000,000 €$ \\
\hline $\mathrm{a}=1.2$ & $\mathrm{a}=2$ \\
\hline$\beta=0.5$ & $\beta=0.1$ \\
\hline $\mathrm{E}=1.0$ & $\mathrm{E}=0.5$ \\
\hline $\mathrm{F}=0.5$ & $\mathrm{~F}=0.1$ \\
\hline $\mathrm{b}=0.7$ & $\mathrm{~b}=0.2$ \\
\hline
\end{tabular}


Hence, for the major industrial accident scenario, the 'rational' risk is calculated to be $136,076 € / \mathrm{yr}$, and the $\mathrm{R}^{*}$ Index equals $442,112 € / \mathrm{yr}$. In case of the domino effect disaster, the rational risk equals $3,600,000 € / \mathrm{yr}$, while $\mathrm{R}^{*}=$ $114,112,310 € /$ yr.

Clearly, using only quantitative (rational) data, the disaster scenario entails the highest risk, but the difference between both scenarios is rather limited, especially when the probabilities are considered as an important decision parameter. However, if moral principles are considered and quantified as displayed in Table 1, this illustrative example shows that the Q\&Q risk index for the domino effect disaster scenario might become much higher than that of the major industrial accident scenario, in turn demonstrating the importance of taking into account principles such as fairness and equity while calculating risk.

Evidently, this given example should only be seen as illustrative to show how moral principles could be integrated into risk calculation, and hence, influence safety decision-making with respect to allocation of safety budgets. The rational risk calculations will always be very important to have a thorough idea of the real situation with respect to risk prioritization, but moral principles can aid to have new insights and a thorough idea of the perceived situation with respect to risk prioritization.

\section{Conclusions}

Current risk calculations are solely based on rational factors such as probabilities and consequences. Based on these rational risk calculations, safety budgets are allocated and decisions are made to lower risk levels. However, this paper argues that risk calculations should take moral aspects such as equity and fairness into account, so that risk acceptability by the public or by the decision-maker can be achieved while deciding on safety budget allocations. Only this way, the next step towards better decision-making with respect to operational safety decisions, can be made.

\section{References}

[1] Center for Chemical Process Safety (2008). Guidelines for chemical transportation safety; security, and risk management. American Institute of Chemical Engineers, Hoboken New Jersey.

[2] van de Poel, I., Fahlquist, J.N. (2013). Risk and Responsibility. In: Essentials of Risk Theory, Roeser, Hillerbrand, Sandin, Peterson (Eds.), Springer, Dordrecht.

[3] Slovic, P. (2000). The perception of risk, Earthscan, London.

[4] Roeser, S. (2006). The role of emotions in the moral acceptability of risk. Safety Science, 44, pp. 689-700.

[5] Roeser, S. (2007). Ethical intuitions about risks. Safety Science Monitor, 11(3), pp. 1-13.

[6] Sunstein, C.R. (2004). Risk and Reason. Cambridge University Press, Cambridge, UK. 
[7] Slovic, P. (2002). The affect heuristic. In: Intuitive judgment: heuristics and biases, Gilovich et al. (eds.), Cambridge University Press, New York.

[8] Wilde, G. (1976). The risk compensation theory of accident causation and its practical consequences for accident prevention. Paper presented at the annual meeting of the Österreichische Gezellschaft für Unfallchirurgies, Salzburg.

[9] Adams, J.G.U. (1985). Risk and freedom: the record of road safety regulation. Transport Publishing Projects, London.

[10] Adams, J.G.U. (1988). Risk homeostasis and the purpose of safety regulation. Ergonomics 31(4), pp. 407-428.

[11] Adams, J.G.U. (1995). Risk. Routledge, Oxon.

[12] Harris, C.E., Pritchard, M.S., Rabins, M.J. (2008). Engineering ethics. Concepts and cases. $4^{\text {th }}$ Ed., Wadsworth, Belmont.

[13] Asveld, L., Roeser, S. (Eds.) (2009). The ethics of technological risk. Earthscan, London.

[14] Hansson, S.O. (2009). Risk and safety in technology. In: Meijers, A. (Ed.). Handbook of the philosophy of science. Philosophy of technology and engineering sciences, vol. 9. Elsevier, Oxford, pp. 1069-1102.

[15] Van de Poel, I., Royakkers, L. (2011). Ethics, technology and engineering. Wiley-Blackwell, London. 